“ C 2019 IEEE. Personal use of this material is permitted. Permission from IEEE must be obtained for all other uses, in any current or future media, including

reprinting/republishing this material for advertising or promotional purposes, creating new collective works, for resale or redistribution to servers or lists, or reuse of any copyrighted component of this work in other works." 


\title{
A 30 Gbps Low-Complexity and Real-Time Digital Modem for Wireless Communications at $0.325 \mathrm{THz}$
}

\author{
Hao Zhang, Xiaojing Huang, Ting Zhang, Jian A. Zhang and Y. Jay Guo \\ Global Big Data Technologies Centre \\ University of Technology Sydney, Australia \\ Emails: \{Hao.Zhang, Xiaojing.Huang, Ting.Zhang, Andrew.Zhang, Jay.Guo\}@uts.edu.au
}

\begin{abstract}
A high-speed wideband terahertz (THz) communication system with low-complexity and real-time digital signal processing (DSP) is presented in this paper. The architectures of baseband platform, intermediate frequency (IF) module and radio frequency $(\mathrm{RF})$ frontend are described. For real-time DSP implementation with affordable field programmable gate array (FPGA) device, some effective strategies are discussed to reduce resource usage and ensure that the clock constraints are met. Adopting these strategies, all physical layer DSP modules are implemented in two FPGAs with more than $300 \mathrm{MHz}$ system clock. The experimental test results using the developed real-time digital modem prototype demonstrate the superb performance for THz wireless communications.
\end{abstract}

Keywords-Low-Complexity, Real-Time, High-Speed, THz System, and FPGA Implementation.

\section{INTRODUCTION}

With ever increasing demand for wireless data transmission, future wireless communication systems will require very high throughput with hundreds of gigabits per second (Gbps) or higher data rate. It becomes obvious that the bandwidth of the future wireless communication systems will increase to tens of $\mathrm{GHz}$, especially for the backhaul systems. Broadband wireless communication systems using the terahertz $(\mathrm{THz})$ frequencies have recently attracted significant interests because of the huge potential applications by opening a super wide untethered bandwidth from $100 \mathrm{GHz}$ to $10 \mathrm{THz}$ for new services.

Due to the relatively lower loss for the carrier frequencies in the $100-300 \mathrm{GHz}$, several $\mathrm{THz}$ communication systems have been reported in recent years. A $2 \times 40$ Gbps wireless communication system using $0.14 \mathrm{THz}$ band oritho-mode transducer is shown in [1]. A fully electronic $90 \mathrm{Gbps}$ one meter wireless link at $230 \mathrm{GHz}$ is presented in [2], and the hardware implementation with parallel sequence spread spectrum (PSSS) modulation using $230 \mathrm{GHz}$ RF frontend is also reported in [3]. With $300 \mathrm{GHz}$ carrier frequencies, a 56-Gbps 16-QAM wireless link is demonstrated in [4]. In these researches, the transmitted signals are all generated by the arbitrary waveform generator (AWG) or signal generator and the received data are analyzed by the digital analyzer or high performance oscilloscope. There is no baseband platform connecting IF module or/and $\mathrm{THz}$ frontend to transmit or receive data from/to medium access control (MAC) layer to form a complete communication system.

There are some experimental systems which connect the baseband platform in either off-line or real-time mode. In [5], a fixed wireless link at $240 \mathrm{GHz}$ carrier frequency is presented with the sampling rate at 64 Giga-samples per second (Gsps). Due to the high sampling rate, the signal processing has to be performed in off-line. A $300 \mathrm{GHz}$ CMOS transceiver for $\mathrm{THz}$ wireless communication is described in [6] which can achieve $20 \mathrm{Gbps}$ with lower sampling rate. A real-time wireless communication system at $0.14 \mathrm{THz}$ is presented in [7], which has throughput of 5 Gbps and a bandwidth of 1.8 GHz respectively.

However, in all of the above mentioned systems, the high-speed data rate and real-time signal processing are not achieved at the same time. In a real-time system, the FPGA is the most important part for the baseband platform to implement the DSP modules, such as encoder/decoder, modulation/de-modulation and channel estimation. There are two typical ways for implementing the DSP modules with FPGA or application specific integrated circuit (ASIC), i.e., the high-level synthesis (HLS) language and the low-level language such as hardware description language (HDL). The HLS language has attracted interests for the system implementation in recent years. Different applications using HLS are shown in [8] and [9]. In both works, the resource usage of the typical cells such as multipliers, block rams (BRAMs) and look-up tables (LUTs) is less than $43 \%$ and the clock speed can only achieve $200 \mathrm{MHz}$. For achieving tens or hundreds of Gbps data rate for wideband wireless communication systems, high-speed system clock such as that of more than 300 $\mathrm{MHz}$ is necessary to reduce the resource usage. Otherwise, it is very difficult to implement all DSP modules in one or two affordable FPGA devices for wideband communication systems. With low-level language, there are a large number of implementations available for typical modules of communication systems, such as low density parity check (LDPC) or Turbo decoder, channel estimation and synchronization. However, there are few systems which are implemented with all physical layer DSP modules for wideband communication links.

In this paper, a real-time digital modem implementation for high-speed $\mathrm{THz}$ wireless communication system is presented. This THz system consists of digital baseband platform, an IF module and commercial RF frontend. Their architectures are described as well. Focusing on the baseband platform, all modules for the transmitter and receiver of the digital modem are presented and some effective strategies which can reduce 
the resource usage and improve the timing performance are described. Adopting these strategies, the implementation of a wideband wireless communication system dealing with some hundreds of Gbps raw data without guarding interval to/from DACs and ADCs becomes possible. The total FPGA resource usage for all DSP modules is provided. The performance of baseband platform, IF module and RF frontend is evaluated and real-time experimental test results are given. Comparison with recently published $\mathrm{THz}$ systems which have baseband processing modules is also made.

The rest of this paper is organized as follows. In Section II, architectures of the proposed $\mathrm{THz}$ communication system as well as the baseband platform, IF module and RF frontend are presented. In Section III, some important strategies for FPGA signal processing implementation are described in details and the resource usage for all DSP modules is provided. Test setup and experimental results are shown in Section IV. Finally, Section V concludes this paper.

\section{System Description}

The high-speed wideband $\mathrm{THz}$ communication system presented in this paper consists of a baseband platform, an IF module and an RF frontend. Fig. 1 shows the system architectures with these three parts. The baseband platform is composed of two DSP units which process $20 \mathrm{Gbps}$ information data from the MAC layer. The baseband platform transmits and receives four baseband signals to and from the IF module by the high-speed digital-to-analog converters (DACs) and analog-to-digital converters (ADCs) with sampling rate at 2.5 Gsps. The four bandpass signals are located at both the upper and lower sidebands of the $15.65 \mathrm{GHz} \mathrm{IF}$ carrier. The IF signal is up-converted and down-converted to/from $325.25 \mathrm{GHz}$ carrier frequency by the 12th harmonics of a local oscillator (LO) of $25.8 \mathrm{GHz}$. The signal frequency conversion from baseband to $\mathrm{THz}$ band is shown in Fig. 2 .

\section{A. Architecture of Baseband Platform}

At the transmitter, typical DSP modules include LDPC encoder, modulation, Tx filter for sample rate conversion (SRC) and pulse shaping, and DAC interface. The encoded bits are mapped into data symbols using 16QAM and the date symbol rate is $1.875 \mathrm{Gsps}$ for each baseband signal. Therefore, the total data rate of four baseband signals is $1.875 \times 4 \times 4$ $=30$ Gbps. The roll-off factor of Tx filter is $1 / 3$ and the bandwidth of modulated signal is $1.875 \times(1+1 / 3)=2.5 \mathrm{GHz}$. After DSP modules, two DACs are adopted for one In-phase and Quadrature-phase (I/Q) modulated baseband signal. The

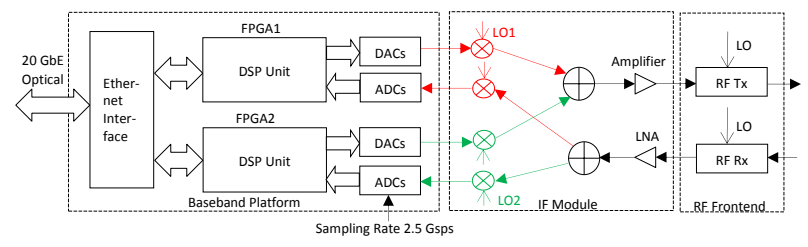

Fig. 1. The system architecture.

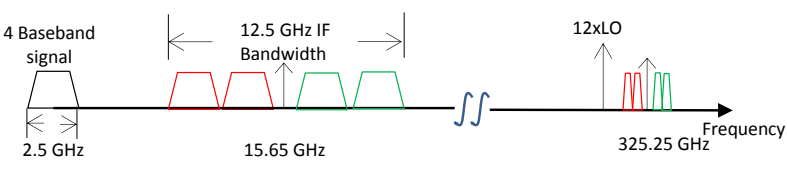

Fig. 2. Frequency conversion from baseband to RF.

signal processing diagram of the transmitter is shown in Fig. 3.

At the receiver, there are synchronization, channel estimation, receive filter, demodulation, decoding and ADC interface modules. For each DSP unit, two I/Q modulated baseband signals are captured by four ADCs sampling at 2.5 Gsps. Following frame synchronization, the captured preamble is used for channel estimation. The coefficients of Rx filters are calculated from channel estimation and the received symbols are retrieved from the Rx filters. The data demapping and LDPC decoding processes are followed after recovering the data symbols. The signal processing block of the receiver is also shown in Fig. 3.

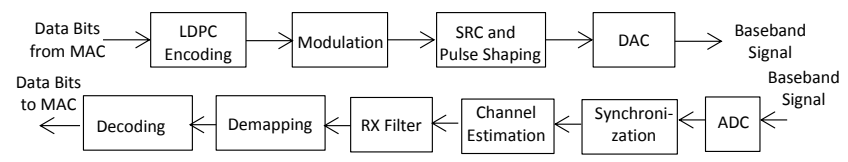

Fig. 3. Transmitter (upper) and receiver (lower) signal processing diagrams.

From Fig. 3, we see that the receiver architecture is simple and highly efficient. There is no feedback between processing modules and each module does not require huge data storage. All modules are run by the parallel pipelined processing. Adopting this kind of simple structure, all modules can be implemented in real-time using affordable hardware. At the same time, the volume and power consumption of the whole system can be easily controlled.

\section{B. Architectures of IF Module and RF}

At the transmitter, the IF module up-converts the I/Q modulated baseband signals generated by the baseband platform to $15.65 \mathrm{GHz}$ IF carrier. There are total 4 channels of baseband signals, each with $2.5 \mathrm{GHz}$ bandwidth. Two of the four bands are first combined to form a $5 \mathrm{GHz}$ bandwidth baseband signal which is further up-converted to the $15.65 \mathrm{GHz}$ IF with only lower sideband. Other two of the four bands are then combined to form another $5 \mathrm{GHz}$ bandwidth baseband signal which is further up-converted to the $15.65 \mathrm{GHz}$ IF with only upper sideband. The lower sideband and upper sideband are finally combined to form a $12.5 \mathrm{GHz}$ bandwidth IF signal centred at 15.65 GHz. A $15.65 \mathrm{GHz}$ pilot frequency is also added for carrier frequency tracking at the receiver. The combined IF signal is amplified by a $30 \mathrm{~dB}$ amplifier and then up-converted to $325.25 \mathrm{GHz}$ RF signal as shown in Fig. 2.

At the receiver, the RF signal is down-converted to 15.65 $\mathrm{GHz}$ IF signal by the 12th harmonics of the LO. Due to the power loss between the transmitter and receiver by the RF frontend, a $30 \mathrm{~dB}$ low noise amplifer (LNA) is connected to 
increase the power of IF signal. The $15.65 \mathrm{GHz}$ IF signal is bandpass filtered to obtain the lower sideband and upper sideband respectively. Each sideband is then down converted to a $5 \mathrm{GHz}$ bandwidth baseband signal, and two channels of the $2.5 \mathrm{GHz}$ bandwidth baseband signals are finally received by the baseband platform. Information data bits are subsequently demodulated by the digital modem.

\section{Digital Signal Processing Implementation}

Baseband platform adopts two Virtex7-690T devices [10] which were introduced in 2010 by Xilinx. This device is popularly used in current industry and academic research. All modules in Fig. 3 are implemented in a single device and one device can deal with 15 Gbps data rate by two baseband signals. Due to the $2.5 \mathrm{Gsps}$ sampling rate of DACs and ADCs, the number of paralled pipelines is 8 and the system clock is $2.5 \mathrm{GHz} / 8=312.5 \mathrm{MHz}$. Due to the huge resources used for all DSP modules, high-speed system clock such as those higher than $300 \mathrm{MHz}$ poses significant challenge for realtime DSP. Therefore, some effective strategies are necessary to reduce the resource usage and optimize timing performance when they are implemented in FPGA.

\section{A. Implementation Strategies}

$\diamond$ Strategy 1: Wide Word-Length Addition Operations

Addition operations are essential when designing the DSP algorithms. The huge number of addition operations with wide word-length occupies lots of LUTs and carry-chains in FPGA. Meanwhile, timing constraints become critical when routing the addition operations, especially with wide word-length. It is not possible to perform lots of operations just in one clock period and hence dividing addition operations into different clock periods is necessary to meet the high-speed system clock timing requirements. Because there are two outputs from one LUT, three additions in one clock period are adopted in our implementation which occupies the same resources as those of two additions in one clock period. Meanwhile, the logic levels between two registers are kept sufficiently simple for the logic routing to meet high-speed clock timing requirements.

\section{$\diamond$ Strategy 2: Block RAM vs Distributed RAM}

When processing all modules, it is necessary to store a certain number of data using the internal memory in FPGA. The BRAM can be used for storing a small number of data. However, timing requirements for the interface of BRAM are difficult to meet, especially when the percentage of occupied BRAM is high and a high-speed system clock is used. Therefore, other kind of memory, the distributed RAM, can be used to replace the BRAM when the size of needed RAM is not very large. Because the distributed RAM is composed of 6input LUTs, it is quite efficient to use distributed RAM for short address memory, especially the RAM with 64 addresses.

\section{$\diamond$ Strategy 3: Resource Sharing}

There are some modules which are parallelly processed continuously without any idle clock, such as LDPC encoder, Tx filters, Rx filters and LDPC decoder. In this case, the resources which are occupied in each clock period can not be shared. However, some modules can be processed over a number of clock periods, such as channel estimation. The resources used for channel estimation can be shared in different clock intervals. For example, as we know, the function of fast Fourier transform (FFT) requires some resources in FPGA. In our design, each IP core of FFT is shared five times. Moreover, some BRAMs and distributed RAMs are re-used by different functions. Although a certain amount of logics for controlling the interface is necessary, the percentage of these additional resources is quite small compared with that of the saved resources.

\section{$\diamond$ Strategy 4: Optimized Precision}

For the digital modem, a high precision is necessary to achieve better performance. However, to achieve high precision, much more resources are required and it becomes more difficult to meet timing requirements. Therefore, optimized algorithms are needed to reduce the resource usage and maintain the performance at the same time, such as the functions of synchronization and $\mathrm{Rx}$ filter. The synchronization consists of coarse timing and fine timing. The coarse timing captures the training sequences in a transmission frame. After this, the fine timing calculates the exact synchronization point from the training sequence segment captured by coarse timing. This implies that the precision of coarse timing can be reduced by using less resources. Although the number of bits for ADCs is as long as 10 , the number of bits used for coarse timing is just 6 in our design. With this reduced precision for coarse timing, the function of synchronization is still achieved and there is no adverse effect on the performance of the whole system. For the $\mathrm{Rx}$ filtering, there are lots of taps in the Rx filter for producing one output symbol. For each symbol, there are the same number of addition operations which can be divided into different levels. Since each addition operation contributes a small portion to the final output, the word length of data can be reduced after each addition level and satisfactory performance can still be achieved for the whole system. The detailed description of the Rx filtering can be found in [11]. After algorithm optimization and implementation, a number of resources are saved and the timing requirements are much easier to be met.

\section{$\diamond$ Strategy 5: Huge Storage Avoidance}

There are four 10 bits ADCs sampling at $2.5 \mathrm{Gsps}$, which means that the throughout of raw data is $4 \times 10 \times 2.5=100$ Gbps. As we know, the internal memory in FPGA is limited and valuable. The system complexity will be increased if the independent external memory is adopted. It is very less efficient to store a huge number of data in any module when performing DSP in real-time. Therefore, avoiding huge storage is necessary for designing and implementing all DSP modules.

$\diamond$ Strategy 6: Specifying Constraints

Placing efficient constraints to some processing logics is essential for meeting timing requirements, especially when a high percentage resource usage occurs and/or some highspeed system clocks are used. Even after optimizing the DSP algorithms and the HDL codes, efficient constraints are still necessary to improve the timing when routing the resource 
in the whole device. In our implementation, some efficient constraints are specified to optimize the routing process and successfully meet timing requirements. Fig. 4 shows some essential constraints applied to the FPGA device. Due to the large number of multipliers used in the whole device, constraints for more than half of the multipliers are applied. In this way, it is possible to place and route a high percentage of multipliers, the number of which is limited for an FPGA device. The green colored seven columns located on the left side of the device show the multipliers which are restrained. Meanwhile, there are nearly twenty Physical Block (Pblock) constraints which are distributed over in the whole device and each of them is decided after some iterations through the synthesis and implementation stages by Vivado tool.

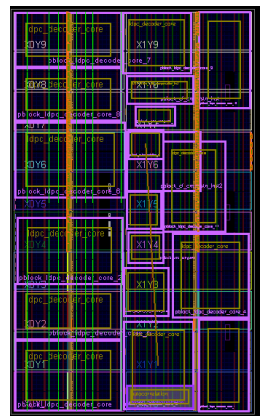

Fig. 4. Essential constraints applied to FPGA device.

\section{B. Implementation Results}

Adopting the strategies presented in Section III.A, the resource usage for all DSP modules and the report of design timing summary are shown in Fig. 5 and Fig. 6 respectively. Although the percentages of LUT and multiplier are $66 \%$ and $63 \%$ respectively, timing requirements for all logics can be still met. Because there are some high-speed clocks for interfaces of ADCs, DACs, GTHs and USB, the percentage of global clock buffer (BUFG) is $62.5 \%$. Due to the necessary replacement of distributed RAM for BRAM, the percentages of LUTRAM and BRAM are about $23 \%$ and $38 \%$ respectively. With a small percentage of BRAM for real-time high-speed system, the difficulty for meeting the timing requirements of the high-speed clocks is effectively reduced. For meeting highspeed clock timing requirements, a large number of flip-flip (FF) registers are necessary and the percentage of FFs is $49 \%$.

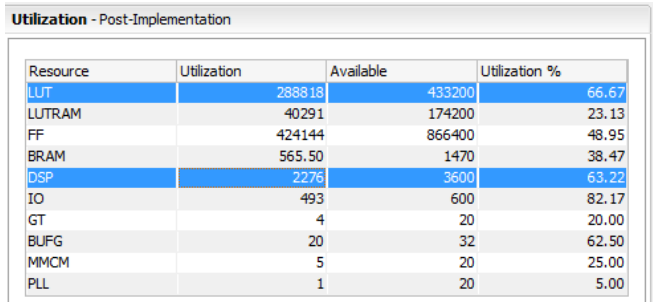

Fig. 5. The report of resource usage.

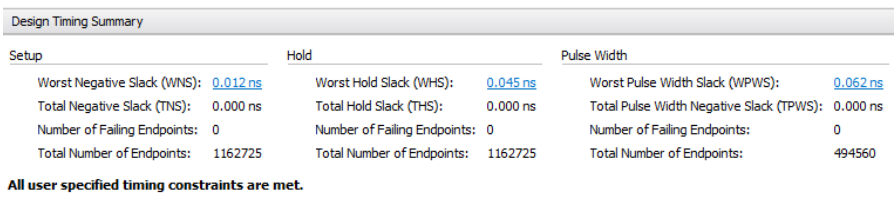

Fig. 6. The report of design timing summary.

\section{Test Setup And ExPerimental Results}

\section{A. Test Setup}

The baseband platform is composed of two FPGA devices, each capable of transmitting and receiving two channels of baseband I/Q signals. The IF transmitter and receiver are connected with the baseband platform and RF frontend via coaxial cable. Commercial RF Tx and Rx frontends are connected by the waveguide. A spectrum analyser is used to monitor the received signal power. A picture of the $\mathrm{THz}$ communication systme testbed is shown in Fig. 7.

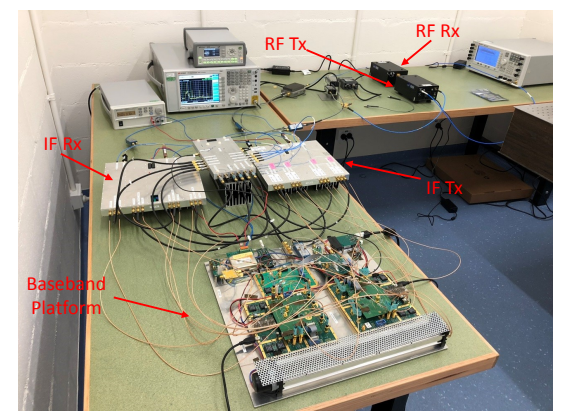

Fig. 7. THz system and test setup.

\section{B. Experimental Results}

Before performing the $\mathrm{THz}$ communication system test, the performance of baseband platform should be verified first. Due to the baseband I/Q modulation architecture, any difference in terms of delay, phase, and amplitude of DACs and ADCs will introduce I/Q imbalance. After DACs and ADCs calibration, the transmitted signal can be looped back via direct DACs and ADCs connection. The error vector magnitude (EVM) of the constellation is $4.0 \%$. From this result, we can ensure that the signal processing produces satisfactory performance for the 16QAM demodulation without IF module and RF frontend.

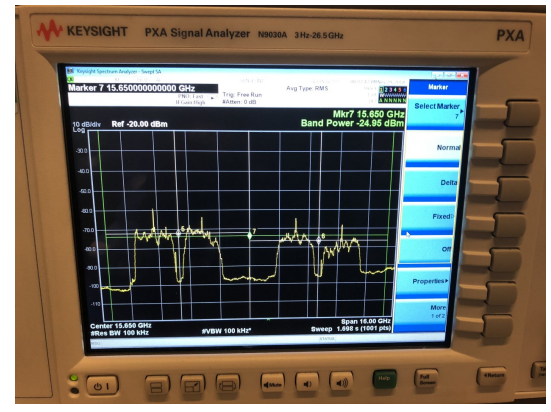

Fig. 8. Four channels of IF signal.

After verifying the performance of baseband platform, the IF module can be integrated with baseband platform. Fig. 8 
shows the four channels of the transmitted IF signals. We can see that there are some LO leakage in each channel. In addition, the power of the four channels are not balanced when transmitting the same signals from baseband platform. These practical impairments introduce some difficulty to the DSP algorithms. Therefore, developing robust algorithms is essential to deal with the impairments from the IF module. After adopting the optimized algorithms, the EVM of received signal constellation for the IF module loopback test is $10.3 \%$.

After testing the performance of the IF module, the whole $\mathrm{THz}$ system can be connected with RF frontend together. The performance is further deteriorated slightly. The EVM of signal constellation for the whole $\mathrm{THz}$ system is $11.6 \%$.

Fig. 9 shows a comparison of the 16QAM constellations among the baseband platform loopback via direct DAC and ADC connection, the IF module loopback and the Thz system connection. With the IF module loopback setup, the measured BER versus Eb/N0 curve is shown in Fig. 10. From this result, we can see that excellent performance is achieved for this wideband system with real-time signal processing.

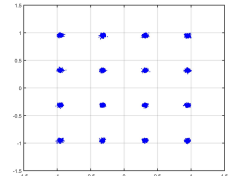

(a) $\mathrm{EVM}=4.0 \%$

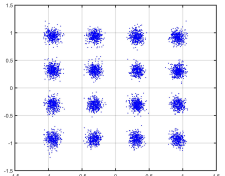

(b) $\mathrm{EVM}=10.3 \%$

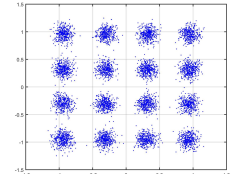

(c) $\mathrm{EVM}=11.6 \%$
Fig. 9. Measured constellations under various loopback tests: Baseband (a), IF (b) and RF (c).

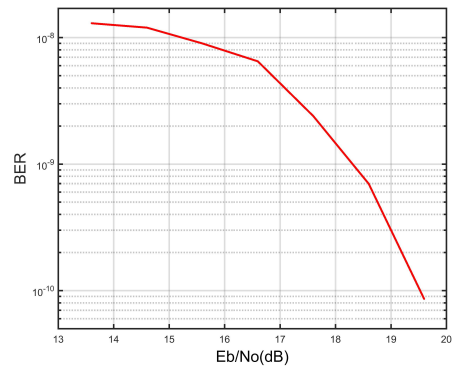

Fig. 10. BER for the IF looped back.

Table I shows a summary of recently published THz communication systems with baseband platforms implemented. The system presented in this paper can achieve 30 Gbps data rate and, more importantly, all the DSP modules are implemented in real-time.

\section{CONCLUSION}

In this paper, a high-speed $\mathrm{THz}$ system with low-complexity and real-time digital signal processing is presented. Some important strategies in FPGA implementation are described for simultaneously achieving low resource usage and meeting timing requirements at high-speed system clocks. The experimental test results using digital and IF hardware prototype verify the excellent performance of the real-time $\mathrm{THz}$ communication system, achieving an EVM of $11.6 \%$. A comparison with $\mathrm{THz}$
TABLE I

Summary of Recently Published THz Communication Systems

\begin{tabular}{|c|c|c|c|c|}
\hline Ref. & {$[5]$} & {$[6]$} & {$[7]$} & This work \\
\hline Year & 2015 & 2018 & 2017 & 2019 \\
\hline EVM & $20.4 \%$ & $12 \%$ & - & $11.6 \%$ \\
\hline Modulation & 8 PSK & 16 QAM & $16 \mathrm{QAM}$ & $16 \mathrm{QAM}$ \\
\hline Symbol Rate (Gbaud) & 21.33 & 5 & 1.25 & $1.875 \times 4$ \\
\hline Sample Rate (Gsps) & 64 & - & 5 & $2.5 \times 4$ \\
\hline Date Rate (Gbps) & 64 & 20 & 5 & 30 \\
\hline Bandwidth (GHz) & 32 & 18.4 & 1.8 & $2.5 \times 4$ \\
\hline Efficiency (Bit/s/Hz) & 2 & 1.08 & 2.78 & 3 \\
\hline Type & Off-line & Off-line & Real-time & Real-time \\
\hline
\end{tabular}

systems published recently and implemented in off-line and/or real-time mode is also made. It is shown that, by adopting the proposed low-complexity design and effective implementation strategies, high-speed wideband wireless communications can be achieved with high performance real-time signal processing.

\section{REFERENCES}

[1] C. Lin, B. Lu, C. Wang, and Q. Wu, "A 240 Gbps wireless communication system using $0.14 \mathrm{THz}$ band oritho-mode transducer," in 2015 40th International Conference on Infrared, Millimeter, and Terahertz waves (IRMMW-THz), Aug 2015, pp. 1-2.

[2] P. Rodrguez-Vzquez, J. Grzyb, N. Sarmah, B. Heinemann, and U. R. Pfeiffer, "Towards 100 Gbps: A fully electronic 90 Gbps one meter wireless link at $230 \mathrm{GHz}$," in 2018 15th European Radar Conference (EuRAD), Sep. 2018, pp. 369-372.

[3] K. KrishneGowda, P. Rodrguez-Vzquez, A. C. Wolf, J. Grzyb, U. R. Pfeiffer, and R. Kraemer, "100 Gbps and beyond: Hardware in the loop experiments with PSSS modulation using $230 \mathrm{GHz}$ RF frontend," in 2018 15th Workshop on Positioning, Navigation and Communications (WPNC), Oct 2018, pp. 1-5.

[4] K. Takano, K. Katayama, S. Amakawa, T. Yoshida, and M. Fujishima, "56-Gbit/s 16-QAM wireless link with 300-GHz-band CMOS transmitter," in 2017 IEEE MTT-S International Microwave Symposium (IMS), June 2017, pp. 793-796.

[5] I. Kallfass, F. Boes, T. Messinger, J. Antes, A. Inam, U. Lewark, A. Tessmann, and R. Henneberger, "64 Gbit/s transmission over $850 \mathrm{~m}$ fixed wireless link at $240 \mathrm{GHz}$ carrier frequency," Journal of Infrared, Millimeter, and Terahertz Waves, vol. 36, no. 2, pp. 221-233, Feb 2015. [Online]. Available: https://doi.org/10.1007/s10762-014-0140-6

[6] S. Hara, K. Takano, K. Katayama, R. Dong, S. Lee, I. Watanabe, N. Sekine, A. Kasamatsu, T. Yoshida, S. Amakawa, and M. Fujishima, "300-GHz CMOS transceiver for terahertz wireless communication," in 2018 Asia-Pacific Microwave Conference (APMC), Nov 2018, pp. 429431.

[7] Q. Wu, C. Lin, B. Lu, L. Miao, X. Hao, Z. Wang, Y. Jiang, W. Lei, X. Den, H. Chen, J. Yao, and J. Zhang, "A $21 \mathrm{~km} 5$ Gbps real time wireless communication system at $0.14 \mathrm{THz}$," in 2017 42nd International Conference on Infrared, Millimeter, and Terahertz Waves (IRMMW-THz), Aug 2017, pp. 1-2.

[8] J. Lei, Y. Li, D. Zhao, J. Xie, C.-I. Chang, L. Wu, X. Li, J. Zhang, and W. Li, "A deep pipelined implementation of Hyperspectral target detection algorithm on FPGA using HLS," Remote Sensing, vol. 10, no. 4, 2018. [Online]. Available: http: //www.mdpi.com/2072-4292/10/4/516

[9] J. Lei, L. Wu, Y. Li, W. Xie, C.-I. Chang, J. Zhang, and B. Huang, "A novel FPGA-based architecture for fast automatic target detection in Hyperspectral images," Remote Sensing, vol. 11, no. 2, 2019. [Online]. Available: http://www.mdpi.com/2072-4292/11/2/146

[10] Xilinx, "FPGA Family," 2010. [Online]. Available: https://www.xilinx. com/support/documentation/data_sheets/ds180_7Series_Overview.pdf

[11] H. Zhang, X. Huang, and Y. J. Guo, "Low-complexity digital modem implementation for high-speed point-to-point wireless communications," in 2018 18th International Symposium on Communications and Information Technologies (ISCIT), Sep. 2018, pp. 16-21. 